\title{
4
}

\section{STAKEHOLDERS, RESPONSABILIDAD SOCIAL EN ECUADOR} Stakeholders, social responsibility in Ecuador.

Christian Morán Montalvo, Leslie Rodríguez Valencia, Mariuxi Torres Olivo, Ana Aguilar Parra, Marta Villalta Borja 


\title{
STAKEHOLDERS, RESPONSABILIDAD SOCIAL EN ECUADOR Stakeholders, social responsibility in Ecuador
}

\author{
Morán Montalvo Christian ${ }^{1}$, Rodríguez Valencia Leslie ${ }^{2}$, Torres Olivo Mariuxi ${ }^{3}$, Aguilar Parra Ana ${ }^{4}$, \\ Villalta Borja Marta ${ }^{5}$ \\ 1-2 Docente tiempo completo de la Facultad de Economía y Ciencias Empresariales de la Universidad de \\ Especialidades Espíritu Santo (UEES) - Ecuador. cmoranm@uees.edu.ec - lprodriguez@ uees.edu.ec \\ 3-4-5 Alumni de la Facultad de Economía y Ciencias Empresariales de la Universidad de Especialidades \\ Espíritu Santo (UEES) -Ecuador. mstorres@uees.edu.ec - anaaguilar@uees.edu.ec - mvillata@uees.edu.ec
}

\section{Resumen}

La importancia de la responsabilidad social empresarial (RSE) radica en el involucramiento y el compromiso de la empresa hacia los diferentes grupos de interés que interactúan. Algunos grupos son afectados a causa de las actividades operacionales de las organizaciones, generando impactos en dimensiones sociales, económicas y ambientales. El enfoque central de la investigación es la determinación de la incidencia de la RSE en los patrones de vida de los grupos que intervienen en la cadena de valor ecuatoriana. En adelante estos son llamados stakeholders. Se precisa como antesala, los hechos y precedentes que marcaron la evolución y participación del modelo de negocio en los diferentes países de Latinoamérica. Sobre esta base, se realiza una revisión conceptual de la responsabilidad social en las empresas, la ISO 26000 y la posición de los stakeholders. El estudio se fundamenta en un análisis comparativo de países como: Chile, Colombia y Ecuador; identificando similitudes de su entorno, particularidades, fortalezas y debilidades en materia de RSE.

Palabras claves: Responsabilidad Social Empresarial, ISO 26000, stakeholders América Latina, Ecuador.

\begin{abstract}
Corporate social responsibility (CSR) is important because companies have involvement and commitment to stakeholders. Stakeholders are affected for the organizational operational activities; this causes an impact on social, economical and environmental dimensions. The focus of the research is to determine the incidence of CSR in living standards involved in Ecuadorian value chain. The investigation defines the facts and precedents for the evolution and participation on businesses model in different Latin American countries. In this context, a conceptual review is performed about social responsibility in businesses, ISO 26000 and the position of the stakeholders. This studio is supported throughout a comparative analysis of countries such as: Chile, Colombia and Ecuador, by identifying similarities, special features, strengths and weaknesses, making reference on CSR.
\end{abstract}

Keywords: Corporate Social Responsibility, ISO 26000, stakeholders, Latin America, Ecuador.

\section{Introducción}

El papel que cumplen las organizaciones en la sociedad ha aumentado considerablemente en los últimos años, convirtiendo a la Responsabilidad Social Empresarial
(RSE) en un modelo clave de gestión. Mediante su aporte libre, espontáneo y voluntario hacia la construcción del mejoramiento social, económico y medioambiental permite el logro de los objetivos organizacionales (Fuertes, Lara, \& Kosacoff, 2006). 
Casado, F. (2006) se refiere a la RSE como una herramienta enfocada en todas las áreas de la compañía generando impactos mediante su operación empresarial de manera directa o indirecta en:
a) la seguridad
b) el entorno laboral
c) el cuidado a los derechos humanos con los grupos de interés
d) la ética empresarial
e) la calidad y reputación de los productos hacia los consumidores
f) las inversiones sociales y medioambientales
g) la productividad
h) la administración de fondos
i) el proceso de toma de decisiones

Esto ocurre independientemente del tamaño, naturaleza y actividad que se ejerza.

Por tanto, el mundo globalizado exige a la empresa que se comporte como un ciudadano corporativo transformador y altamente dinámico con requerimientos competitivos cada vez mayores. Del mismo modo, que use la RSE como un medio para lograr una sociedad más equitativa, justa, y sustentable. He entonces la importancia de que prevalezca un entorno adecuado y una gestión íntegra que permita un estilo de vida digno, así como el fortalecimiento de relaciones saludables con los stakeholders (Roser, 2005).

La revisión literaria empieza con el estudio de los siguientes términos: RSE, ISO 26000 y stakeholders. Luego se prosigue a mostrar el escenario latinoamericano frente a la RSE mediante un análisis comparativo entre países como Ecuador, Chile y Colombia. Este estudio, específicamente, pretende evidenciar el contraste de la evolución de la RSE en estos países describiendo sus prioridades y la posición de sus stakeholders frente a la empresa. Por otro lado, se busca destacar el soporte de la institución pionera y promotora de RSE en América Latina, al igual que su participación e involucramiento activo en debates y reuniones internacionales.

Una vez analizada la situación actual de la RSE de manera regional en América Latina, se dará a conocer la realidad de las empresas ecuatorianas frente a temas de responsabilidad corporativa. Asimismo, se describe un caso práctico de implementación de iniciativas a favor del desarrollo sostenible ejecutado por la empresa líder de la industria de bebidas alcohólicas, Cervecería Nacional S.A.

La finalidad de la presente investigación es mostrar una visión más clara de la RSE y su desarrollo en el mercado ecuatoriano. El propósito es que el contenido contribuya a emitir juicios de valor más concretos que generen decisiones acertadas por parte de las organizaciones. La interacción constante con los stakeholders debe funcionar en aras de generar el crecimiento económico y el fortalecimiento de la cadena productiva del país.

\section{Revisión Literaria}

Este trabajo tiene como bases conceptuales los términos: RSE, ISO 26000 y stakeholders; mediante la exploración de aportes científicos de diferentes autores.

Según, Méndez (2007) un análisis etimológico de la palabra responsabilidad proveniente del vocablo responsum en latín, representa el verbo responder, por lo cual se infiere que la RSE conlleva a dar respuestas a la sociedad desde la perspectiva empresarial.

Por su parte, la Organización de Normalización Internacional (ISO) vincula a la RSE con base en la sostenibilidad en tres dimensiones que son: la económica, medioambiental y social (Corrales, 2011). Fernández (2011) expresa que al asociar la RSE con la dimensión económica propone que las compañías enfrenten el reto de cambiar la manera de producir bienes y servicios uniendo el progreso económico con el uso adecuado de los recursos, lo cual contribuya positivamente al ecosistema y a la reducción de costos empresariales así como a la generación de plazas de empleos y la promoción del consumo racional.

En ese sentido, la empresa socialmente responsable (ESR) debe enfocarse en la dimensión social, no solo a través de la creación de fuentes de trabajo, sino en el desarrollo de iniciativas sostenibles que influyan en un mejor estilo de vida de los colaboradores y sus familias. La comunidad y demás grupos de interés también deben verse beneficiados mediante la creación de centros de salud, de educación y formación técnica de la cadena de valor (Guibert, 2009).

Siendo el medio ambiente, la fuente de recursos naturales que desde el ámbito económico es el determinante para los asentamientos humanos y las decisiones empresarialeses, es de vital importancia que se realice mejoras en el tratamiento de procesos de producción para llegar a la ecoeficiencia y a la maximización del uso adecuado de insumos y recursos (Bangochea, 2010).

Con la finalidad de orientar y fortalecer las operaciones organizacionales, de manera que sean socialmente responsables, el organismo internacional ISO publicó el 1 de noviembre del 2010, la Norma ISO 26000. De acuerdo al secretario general de ISO, Steele (2010) considera que la "ISO 26000 es una poderosa herramienta que ayudará a las organizaciones a pasar de las buenas intenciones a las buenas acciones", claramente no pretende ser un certificado de gestión empresarial, sino más bien una guía de directrices y pautas para desarrollar el modelo en la organización. Bajo este enfoque, la ISO contiene siete materias 
fundamentales que son la gobernanza de la organización, los Derechos Humanos, las prácticas laborales, el medioambiente, las prácticas justas de operación, los asuntos de consumidores, la participación activa y el desarrollo de la comunidad (Alonso, 2006).

Por su parte, los stakeholders de acuerdo a Freeman, se refiere a "un grupo o individuo que puede afectar o es afectado por la realización de los objetivos de la organización" (Payne \& Calton, 2004). Por añadidura, se afirma que los stakeholders toman una posición activa y legítima ante los aspectos sustanciales de la empresa porque mediante su accionar afecta a las decisiones y actividades de la misma (Navarro, 2012). Por tanto y como se observa, se puede afirmar que los stakeholders interactúan en lo que se considera una contabilidad integral de todos los procesos en el cual la empresa debe cumplir con el deber de rendir cuentas a la sociedad, buscando la transparencia y reconocimiento ante el mercado (Cooper, 2004).

Según Navarro (2012) los stakeholders de acuerdo con su cercanía y actividad en la empresa se pueden identificar en las dos siguientes categorías:

a) Stakeholders internos: es el grupo de intervinientes ligados directamente a la empresa sean estos: accionistas, socios, directivos, colaboradores, sindicatos y proveedores o socios estratégicos, etc.

b) Stakeholders externos: es el grupo no vinculado de manera directa a la empresa como son consumidores, gobierno, competencia, grupos de presión, etc.

\subsection{La RSE y su evolución en Latinoamérica}

Conscientes de las oportunidades y desafíos que enfrenta el Ecuador ante la RSE, se analiza comparativamente la evolución en materia de desarrollo sostenible en Latinoamérica mediante la investigación de aportes científicos de diferentes autores como Alonso (2006), Vives (2008), Fundación Korin (2011). Conjuntamente se trabaja con la cooperación de estudios realizados por instituciones promotoras de la RSE como Business School, Cooperación Alemana al Desarrollo GIZ, Ekos Negocios, y la Cámara de Comercio Ecuatoriano Americano.

En Latinoamérica, la RSE empieza a surgir por la necesidad de vincular a la empresa con la comunidad desde el siglo XVI al XIX donde se promovían acciones de ayuda social motivadas por los valores éticos y religiosos de los propietarios de pequeñas y medianas empresas (Pymes) que generalmente eran de índole familiar (Gutierrez, Avella, \& Villar, 2006). Acto seguido, en el siglo XX se realizaron los primeros acuerdos internacionales $\mathrm{y}$ hechos que marcaron la historia mundial - como la Declaración Universal de los Derechos Humanos, la Declaración de la Organización
Internacional del Trabajo, las directrices dictadas por las Naciones Unidas a favor del consumidor, el nacimiento de la globalización y el auge de la tecnología de información y la comunicación (TIC) - logrando de esta manera que la responsabilidad social sea la nueva preocupación organizacional que traspase fronteras tomando mayor fuerza sobre todo en las dos últimas décadas (AVINA \& Korin, 2011).

Según Riechmann y Naredo (1995), uno de los organismos promotores de la RSE es la Organización de las Naciones Unidas, quienes en 1987 mediante la creación de la Comisión Mundial del Medio Ambiente y el Desarrollo, dio un gran aporte a la historia mundial a través la publicación del Informe de Brundtland o "Nuestro Futuro Común". El estudio estuvo enfocado en la construcción del desarrollo sostenible, planteando la reflexión hacia la búsqueda de la satisfacción de las necesidades del presente, sin afectar a las futuras generaciones; determinando que los grandes problemas de desigualdad y pobreza en la sociedad se deben a la falta de visión social al momento de alcanzar la prosperidad económica empresarial.

Por otro lado, en la década de los noventa, la globalización impulsaba a pasos agigantados la competitividad en las empresas alineadas a normas laborales y medioambientales (Altvater \& Mahnkopf, 2002). Sin embargo, se evidenció mediante la TIC, los comportamientos no éticos e irresponsables de grandes corporaciones para lo cual decidieron no seguir en la línea tradicional del negocio que se basaba únicamente en el precio, innovación y sobre todo publicidad (Morales, 2012).

En el año 1992 se instaura la Cumbre de la Tierra en Rio de Janeiro, convocada por las Naciones Unidas donde se planteó en la agenda mundial, las preocupaciones de carácter ambiental como la problemática del calentamiento global y la necesidad urgente de crear políticas y mecanismos que permitan mitigar los daños causados en la naturaleza, reuniendo 172 naciones y 2400 organizaciones civiles. A consecuencia de la Cumbre de la Tierra se consolida en años posteriores el Consejo Empresarial Mundial para el Desarrollo Sostenible (por su nombre en inglés: World Business Council for Sustainable Development), impulsando la formación de redes y organizaciones a favor de la RSE (Carneiro, 2004).

Siendo así que en Latinoamérica se empieza a sentir la tendencia arrolladora de la RSE. Con mayor incidencia en países como Brasil, Chile y México quienes lideran a nivel regional, seguido de Colombia, Argentina y Centroamérica. Para entonces, Ecuador forma parte del tercer grupo del continente donde su evolución no ha permeado en lo nacional debido a que su implementación se presenta notoriamente en casos aislados como empresas multinacionales, segmentos de exportadoras, 
empresas extractivas privadas y nacionales y de manera escasa en las pymes (Morales, 2012).

Latinoamérica está pasando por un proceso de transición y cambios en el sistema económico, político y social, teniendo una estructura empresarial comúnmente conformada por un $99,8 \%$ por micro, pequeñas y medianas empresas que en general son de tipo familiar, mientras que el $0,2 \%$ representa a grandes corporaciones. Consecuentemente de esa estructura dominante donde se desarrolla el mercado de la RSE, se ha identificado que los avances en el tema no son coherentes ni precisos en continentes como el americano con el europeo (Jáuregui, 2008).

Según Vives (2008) las variables condicionantes más destacadas y comunes en América Latina respecto al mercado de RSE nacen de las economías emergentes de países de la región, sistemas políticos inestables y corruptibles, falta de institucionalidad del gobierno corporativo, subdesarrollo de los stakeholders, fallos en el suministro de servicios, pobreza, desigualdad, falta de interés en la prioridad de la sostenibilidad y falta de capacitación al personal.

Las investigaciones realizadas por Fundación AVINA \& Korin (2011) y Alonso (2006) con el apoyo de la Comisión Económica para América Latina y el Caribe (CEPAL), sirven de fuente para realizar un análisis comparativo de la situación actual en materia de RSE de Chile, Colombia y Ecuador canalizando el primer objetivo del estudio.

El análisis identifica las cualidades similares de los tres países, así también las particularidades de cada nación. Siendo así, Chile uno de los países líderes en el avance de la RSE en Latinoamérica se determinó que el concepto se asocia principalmente en la calidad de vida de sus colaboradores, mientras que Colombia tiene muy arraigada la filantropía tradicional y es originada por las preocupaciones del Estado. Por su parte, Ecuador es uno de los países con menor permeabilidad nacional del modelo, no obstante, cuenta con la participación de organizaciones y redes formadas por la sociedad civil y las asociaciones empresariales, existiendo un común denominador en los tres países de incursionar el empresariado como un ente socialmente responsable que se genera a partir de los requerimientos de mercados internacionales y de las demandas de la globalización (Alonso, 2006).

Otro de los estudios analizados fue el realizado por Fundación AVINA \& Korin (2011) donde expresa la participación de los tres países en foros, discusiones y debates de la ISO 26000 considerando que Chile representa el $13,30 \%$ en intervención con un total de 50 participantes, Colombia tiene el $7,71 \%$ con 29 participantes y Ecuador el 1,6\% con 6 participantes siendo el país con menos intervención y presencia. Asimismo, este organismo promotor de la RSE generó mayor soporte y financiamiento en iniciativas en Chile, Ecuador y en último lugar a Colombia.

De acuerdo a sus hallazgos, Alonso (2006) manifiesta que en cada país tiene grupos de interés con mayor presión hacia las empresas como es el caso de Chile que tiene mucha presencia y relevancia de los trabajadores, consumidores y de las ONG. En Colombia, el Estado es el grupo de interés con mayor representación lo cual se debe a la situación de conflicto que vive el vecino país En Ecuador la presencia del Estado es sumamente frágil respecto a la RSE y no hay una verdadera organización a favor de colaboradores y consumidores.

\section{Tabla \# 1. Países latinoamericanos frente a la Responsabilidad Social Empresarial}

\begin{tabular}{|c|c|c|c|}
\hline \multirow[t]{2}{*}{ Variables } & \multicolumn{3}{|c|}{ Países latinoamericanos participantes en el análisis } \\
\hline & Chile & Colombia & Ecuador \\
\hline Estado de la RSE & $\begin{array}{l}\text { La RSE se asocia con calidad de } \\
\text { vida de los colaboradores. Es uno } \\
\text { de los países líderes en la región. }\end{array}$ & $\begin{array}{l}\text { La RSE está asociada con la } \\
\text { filantropía tradicional. }\end{array}$ & $\begin{array}{l}\text { La RSE no es un tema } \\
\text { universal solo se ha } \\
\text { presentado casos aislados } \\
\text { de su aplicación. }\end{array}$ \\
\hline $\begin{array}{lccc}\text { ¿A qué se debe } & \text { la } \\
\text { implementación } & \text { de } & \text { la } \\
\text { RSE? } & & \end{array}$ & $\begin{array}{l}\text { Requerimientos de mercados } \\
\text { internacionales, Consumidores } \\
\text { extranjeros y presiones sociales. }\end{array}$ & $\begin{array}{l}\text { La RS está posicionada en la } \\
\text { agenda de gobierno por la } \\
\text { privatización. Por exigencias de } \\
\text { mercados internacionales. }\end{array}$ & $\begin{array}{l}\text { Requerimientos de } \\
\text { mercados internacionales } \\
\text { y la globalización. }\end{array}$ \\
\hline $\begin{array}{l}\text { Participación en debates } \\
\text { de ISO } 26000 \text {. }\end{array}$ & $13,30 \%$ con 50 participantes. & $7,71 \%$ con 29 participantes. & $1,60 \%$ con 6 participantes. \\
\hline $\begin{array}{lll}\text { Posición de } & \text { los } \\
\text { stakeholders. } & & \end{array}$ & $\begin{array}{l}\text { Las ONG, los consumidores y los } \\
\text { trabajadores tienen gran } \\
\text { representación frente a materia de } \\
\text { RSE mientras que el Gobierno se } \\
\text { presenta como el grupo más débil }\end{array}$ & $\begin{array}{l}\text { Las ONG y el gobierno tienen } \\
\text { gran representación en de RSE } \\
\text { mientras que el grupo de } \\
\text { consumidores y trabajadores } \\
\text { tienen posiciones débiles. }\end{array}$ & $\begin{array}{lrr}\text { El Estado frente a la RSE } \\
\text { es frágil, } & \text { los } \\
\text { consumidores y } & \text { los } \\
\text { colaboradores r no } & \text { están } \\
\text { organizados. } & & \end{array}$ \\
\hline
\end{tabular}




\begin{tabular}{|c|c|c|c|}
\hline $\begin{array}{l}\text { Soporte y financiamiento } \\
\text { de Fundación AVINA }\end{array}$ & $\begin{array}{l}\text { La contribución económica } \\
\text { asciende a más de USD } 1.000 .000 \\
\text { con } 20 \text { iniciativas apoyadas. }\end{array}$ & $\begin{array}{l}\text { La contribución económica es } \\
\text { menor a los USD } 500.000 \text { con } \\
\text { menos de } 10 \text { iniciativas } \\
\text { apoyadas. }\end{array}$ & \begin{tabular}{lrr} 
La & \multicolumn{2}{r}{ contribución } \\
económica & es de & USD \\
1.000 .000 & con & 10 \\
iniciativas apoyadas. &
\end{tabular} \\
\hline
\end{tabular}

Fuente: De "En busca de la sostenibilidad. El camino de la RSE en América Latina y la contribución de la Fundación AVINA", por M. Korin y Fundación AVINA, 2011. De "Avances en discución sobre la ISO 26000 en América Latina: antecedentes para apoyar el proceso ISO en la Región" por V. Alonso, 2006.

\subsection{La RSE en Ecuador}

Los últimos años para Ecuador, la agenda de la RSE ha sido muy dinámica e interactiva debido al despertar del interés de empresarios, organizaciones y sociedad civil respecto a la temática, desarrollándose numerosos congresos, debates, servicios profesionales de consultoría y reportes de sostenibilidad. De esta manera, organizaciones promotoras de la RSE en el país como IDE Business School, Fundación AVINA, Cooperación Alemana al Desarrollo GIZ, Ekos Negocios, Cámara de Comercio Ecuatoriana Americana, Consultor Apoyo y el Ministerio de Coordinación de la Producción, Empleo y Competitividad realizaron un estudio que fue publicado en el año 2012 sobre la perspectiva de la responsabilidad social en los negocios.

Para ello, se trabajó de acuerdo con la postura del consumidor con 1052 participantes entre hombres y mujeres mayores de 18 años, enfocados en ciudades como Quito, Guayaquil, Cuenca, Sucumbíos y Orellana. El análisis de alcance nacional, determinó que 7 de cada 10 consumidores tienen escasa e imprecisa información respecto al concepto de la RSE, no obstante, el 98\% de este grupo consideran que las ESR son de gran importancia e influye en el proceso de decisión de compra, mientras que el $85 \%$ de los consultados opinan que la ESR supera a una que no lo es por lo cual el $94 \%$ estaría dispuesta a dejar de consumir la marca actual por un producto o servicio elaborado y ofertado por una organización socialmente responsable. Según, los encuestados participantes del estudio al que estamos haciendo referencia, el $86 \%$ consideran que la RSE debe ser aplicada de manera obligatoria en todas las organizaciones por lo cual debería existir una base legal por parte del Estado que lo promueva, exija y controle.

Es interesante destacar que la Constitución Política del Ecuador reformada en el año 2008, impulsa el régimen del desarrollo mediante sus principios generales que establece directrices del buen vivir, construyendo un sistema económico sostenible, justo, y respetuoso hacia la naturaleza, así como también se vela por mejores niveles de vida de la comunidad. Sin embargo, este documento legal no define mecanismos efectivos alineados al incentivo y control de la RSE debido a que no hay una determinación del concepto en el texto sobre empresas socialmente responsable.
Por su parte, la reconocida firma auditora, Deloitte, mediante su informe anual de Percepción de la Responsabilidad Corporativa y Sostenibilidad (RC\&S) revela la postura del empresario en materia de RSE, mediante las encuestas efectuadas y analizadas de julio a agosto del año en curso. En la encuesta efectuada sobre RC\&S participaron 62 empresarios de organizaciones ecuatorianas quienes afirmaron en un $66 \%$ que la responsabilidad corporativa está ligada como consecuencia al rendimiento económico y financiero de la organización con un efecto multiplicador del valor agregado, buena reputación y atención por parte del mercado y bienestar hacia sus stakeholders.

Las empresas partícipes de esta observación atienden en un $21 \%$ como público de mayor dedicación e interés a sus colaboradores, porque todo empieza desde la vertiente de la cultura organizacional dejando como último plano, lamentablemente, las relaciones con los proveedores. Los consultados de la encuesta en mención, indicaron que la sostenibilidad organizacional está siendo administrada en un $55 \%$ con el apoyo de la alta dirección, teniendo la responsabilidad la gerencia general con un decrecimiento de 6 puntos frente al año 2013; mientras existe una clara tendencia de la creación de un departamento focalizado exclusivamente en RSE con un crecimiento del $6 \%$ de los resultados del año anterior (Deloitte, 2014).

Ecuador forma parte de la red de mapeo de organizaciones promotoras de RSE de América Latina con 40 organizaciones conformadas en su mayoría por las asociaciones civiles y las organizaciones empresariales para fomentar la RSE en el medio. La red de mapeo de promotores de RSE en América Latina, tiene una herramienta digital de fácil acceso y de manera gratuita mediante su sitio web ${ }^{1}$ para utilidad del público en general, contando con la participación de organismos que se encargan de la formación, divulgación, capacitación, asesoramiento e incentivo de la temática.

La intervención de las instituciones que están enmarcadas en el desarrollo de la RSE tiene una gran responsabilidad y desafío por cumplir frente a las carencias sociales que existen como son la desigualdad, la corrupción, el desempleo, los problemas ambientales y la pobreza (Correa, Flynn, \& Amit, 2004). Siendo la

\footnotetext{
${ }^{1}$ Sitio Web Red de mapeo de promotores de RSE en América Latina: www.mapeo-rse.info
} 
pobreza, el problema social más preocupante del Estado que de acuerdo con el análisis cuantitativo realizado en el último trimestre del presente año por el Instituto Nacional de Estadísticas y Censos INEC (2014), revela un incremento porcentual desfavorable para el país con relación al año 2013 que era de $23.69 \%$ y a la actualidad es de 24, 53\%, evidenciando que la brecha de la inequidad tuvo un leve crecimiento.

\section{Metodología}

En primer lugar, se estableció una revisión literaria bajo términos como RSE, ISO 26000 y stakeholders para conocer la importancia y accionar en las operaciones empresariales. Posteriormente, se analizó el escenario de la región latinoamericana frente a la RSE a través de una matriz comparativa entre países de la región como son Chile, Colombia y Ecuador. En los tres casos, se identificaron las particularidades y similitudes en contexto al estado de la RSE, su implementación, la posición de los stakeholders, la participación en debates de la ISO 26000 y el soporte de Fundación AVINA a iniciativas de cada país. Se realizó un estudio del escenario de la RSE en Ecuador evidenciando sus fortalezas, debilidades y desafíos mediante la observación efectuada por organismos de gran credibilidad en el país, así como el reconocimiento de la participación activa de organizaciones que forman parte de la red promotora de RSE ecuatoriana.

Consecutivamente, se presenta el estudio descriptivo de los avances y mecanismos a favor del desarrollo sostenible de la empresa Cervecería Nacional S.A. Cabe destacar, que a través de este caso no se trata de mostrar una determinación estricta o una norma de aplicación universal en el Ecuador, pero sí de contar los elementos de caracterización de la empresa respecto a las prácticas socialmente responsables.

\section{Resultados}

\subsection{Cervecería Nacional y sus ejes de acción en el desarrollo sostenible}

De acuerdo con la investigación realizada mediante el presente estudio se revela la práctica a favor del desarrollo sostenible, base de la RSE, de una reconocida empresa multinacional llamada Cervecería Nacional S.A. (CN) perteneciente al grupo SAB MILLER, compañía que tiene 127 años de trayectoria en la industria de bebidas alcohólicas en el país con un portafolio amplio de productos altamente aceptados por el mercado ecuatoriano revelando de esta manera las iniciativas y los mecanismos ejecutados para alcanzar la rentabilidad social, ambiental y económica (Cerveceria Nacional, 2014). Cervecería Nacional S.A. centra su atención en cinco prioridades del desarrollo sostenible con un alto compromiso hacia los stakeholders que son: la cultura de moderación de consumo cervecero, el cuidado y desarrollo de la comunidad para mejorar los niveles de vida, la protección del medio ambiente, los procesos productivos de clase mundial y el mundo productivo con el uso mesurado de los recursos (Cervecería Nacional S.A., 2014).

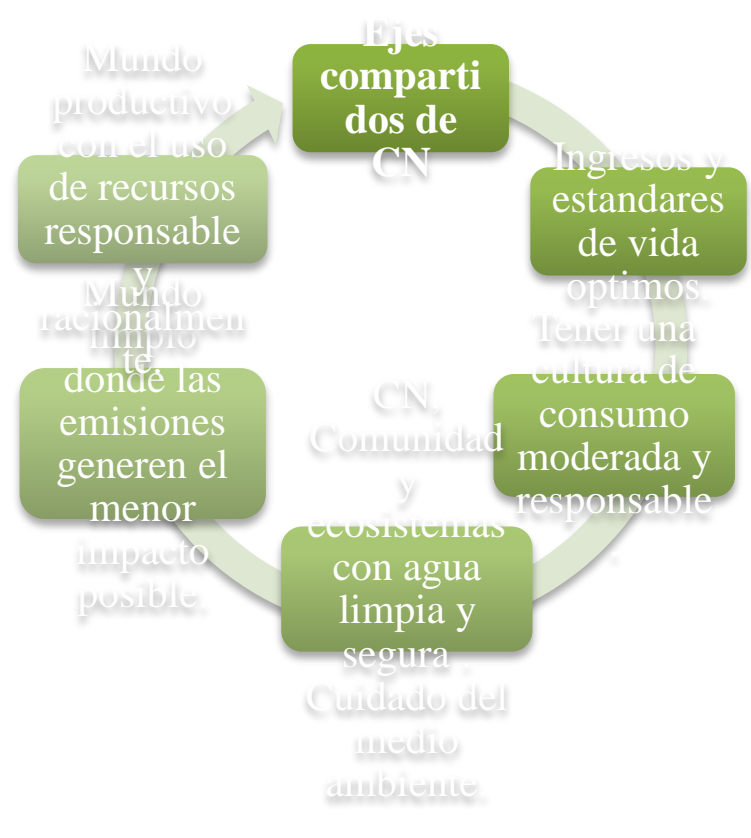

C. Morán/L. Rodríguez/M. Torres/A. Aguilar/M. Villalta 
Figura 1. Ejes compartidos del Desarrollo Sostenible de Cervecería Nacional S.A.

Fuente: Elaboración propia basada en el portal web http://www.cervecerianacional.ec/desarrollo-sostenible.php.

\subsection{Cervecería Nacional S.A. y su dimensión socioeconómica}

La empresa se ha convertido en un ente impulsador del bienestar, creando fuentes de trabajos directos e indirectos, manteniendo una amplia cadena de valor entre 3000 proveedores que facilitan la materia prima para la producción donde participan 2040 colaboradores obteniendo el producto final que es comercializado por 133000 socios y distribuidos hacia la venta final a los consumidores generando rentabilidad a 1129 accionistas y hogares de la red productiva, dinamizando la economía mediante los impuestos recaudados que facilitan la inversión social (Cerveceria Nacional, 2014).

$\mathrm{CN}$ es promotor del desarrollo de programas hacia la comunidad generando destrezas, habilidades y conocimientos que les permitan mejorar sus niveles de vida. El proyecto de desarrollo comunitario Santa Inés es una muestra de aquello donde han participado 300 habitantes que viven en los alrededores de la Planta $\mathrm{CN}$ Cumbayá en talleres de emprendimiento y cursos de dibujo, pinturas, fachadas gracias al soporte e involucramiento del Municipio del Distrito Metropolitano de Quito y Fundación Esquel. Se ejecutó en este programa las siguientes etapas:

a) Socialización a la comunidad hacia la organización a favor de un mejor estilo de vida.

b) Aprendizaje, desarrollo de destrezas y habilidades de interacción de la comunidad con otros actores.

c) Puesta en marcha a través de una comunidad fortalecida que realiza acciones a favor del ambiente, procesos productivos, liderazgo y emprendimiento.

Asimismo, según el Informe CN "Haciendo la diferencia" (2011) se busca mediante el programa Siembra Futuro, la capacitación y formación de tenderos que ofrezcan productos para incrementar sus estándares de vida e ingresos, considerando temas referentes al servicio al cliente, registros de ventas, presentación del producto, liderazgo, promoción del consumo moderado y responsable (prohibida la venta a menores de edad). Actualmente a nivel nacional se pueden beneficiar 6700 tenderos a los cuales se les enseña técnicas y directrices que los permitan ser vendedores responsables, sostenibles, excelentes y líderes.
Otro aporte señalado por $\mathrm{CN}$ en su sitio $\mathrm{web}^{2}$ es la gran iniciativa realizada en Pascuales, mediante el programa Zona Haciendo la Diferencia Pascuales, efectuado a favor de la comunidad desarrollándose en los alrededores de Pascuales donde se ofrece a 200 niños y niñas actividades recreativas, deportivas y el fortalecimiento de valores así como la capacitación a 50 mujeres en destrezas como elaboración de bisuterías, accesorios y chocolatería.

$\mathrm{El}$ proyecto Siembra Cebada realizado por la $\mathrm{CN}$, logra seguir manteniendo la siembra del tradicional cultivo de la cebada otorgando a los participantes semillas certificadas, fertilizantes e insumos agrícolas, así como capacitación, asesoramiento y seguimiento, favoreciendo la cosecha óptima que asegure la comercialización a un precio justo de la producción. Promueve el desarrollo de negocios inclusivos que permitan minimizar la pobreza y mejorar la calidad de vida de las comunidades intervinientes en el proyecto en las provincias de Imbabura, Carchi y Pichincha ayudando a 650 agricultores y 3250 personas beneficiadas que integran sus familias. El objetivo del programa busca desarrollar la cadena de valor a largo plazo, con la visión de que estos pequeños agricultores se conviertan en proveedores locales que pueden ser incorporados en la operación de la organización (Cervecería Nacional S.A., 2011).

Los consumidores son parte esencial de la operativa del negocio por lo tanto $\mathrm{CN}$ ha impulsado la campaña "Disfruta con moderación" que según el informe $\mathrm{CN}$ Haciendo la Diferencia (2011) se revela las iniciativas emprendidas por la empresa en centros de entretenimientos y diversión en ciudades como Quito, Guayaquil y Cuenca a favor del consumo moderado cuyo objetivo es hacer conciencia entre los consumidores para evitar el consumo irracional y la venta a menores de edad de las bebidas alcohólicas que comercializa la empresa. Existe preocupación y se prioriza la prevención de que el consumidor corra riesgos de accidentes de tránsitos manejando bajo los efectos del alcohol.

\subsection{CN y su dimensión medio ambiental}

De acuerdo al Informe CN Haciendo la Diferencia (2011), la empresa realiza campañas de reciclaje, de uso responsable de los recursos utilizados en la organización

\footnotetext{
${ }^{2}$ Sitio Web Cervecería Nacional S.A.: http://www.cervecerianacional.ec/pr-zona.php
} 
y de cuidado al medio ambiente, empezando desde casa adentro donde se desarrollan acciones a favor al cuidado de la naturaleza. $\mathrm{CN}$ tiene en la planta Guayaquil, 40 estaciones de Reciclaje y en la planta Quito, 16 estaciones para que los colaboradores tengan una cultura del uso y depósito adecuado de los desechos. Las actividades que se realizan externamente en la empresa con el soporte de instituciones como Recynter y Owen Illinois desde el 2008 es el Reciclaje de Botellas en ciudades como Guayaquil, Quito, Playas, Salinas, Atacames, San Cristóbal y Santa Cruz con una red de 93 estaciones y 7 estaciones móviles que se transportan a lugares de esparcimiento y diversión a nivel nacional, en el camino se busca realizar en su gestión la reutilización del 95\% de envases en su producción y caminar hacia su meta principal que es tener cero desperdicios.

Las plantas de tratamiento de aguas residuales buscan eliminar todo elemento contaminante del $100 \%$ del agua utilizada en los procesos productivos para así obtener agua limpia que se emplee para otra funciones o actividades como jardinería y limpieza. Asimismo, se usa de manera racional la energía a través de mecanismos como la evaporación al vacío implementado en las plantas de producción de Quito y Guayaquil; donde utilizan de forma óptima la energía térmica generada por la cocción de los productos lo cual es almacenado en dispositivos especiales generadores de energías nuevas que es direccionada a la producción y el uso de paneles solares que permiten producir electricidad (Cerveceria Nacional, 2014).

\subsection{CN y la cultura de la Ética}

La organización impulsa la ética como una responsabilidad de todos, reportando de manera pública su desempeño financiero y de todas las actividades alineadas al Código de Ética y Manifiesto de alcohol. Cabe destacar que los grupos de interés como colaboradores, proveedores, distribuidores y franquiciados, tienen acceso a información económica anual de la empresa con el fin de promover el respeto, admiración y transparencia de la operación, así como tienen la libertad de denunciar acciones que atenten contra sus principios. Los colaboradores son periódicamente informados sobre normas y principios que rigen el Código de Ética, así como de los beneficios y oportunidades de desarrollo profesional que puedan obtener (Cervecería Nacional S.A., 2011). Cervecería Nacional con todas estas acciones de apoyo al Desarrollo Sostenible se ha convertido en una de las empresas de más trayectoria del país y ha obtenido reconocimientos internacionales y nacionales publicados en su portal web como:

a) Mejor Práctica de Responsabilidad Social Empresarial 2013 de América Latina en la categoría Alianzas Intersectoriales por el Centro Mexicano de Filantropía (CEMEFI).

b) Primera empresa extranjera en conseguir el primer lugar de los Premios Perú 2021 en la categoría de Mejor Práctica de Responsabilidad Social y Desarrollo Sostenible.

c) Finalista en la categoría Innovación Social de la Empresa de la XVII Edición de los Premios CODESPA, de España.

d) Certificación de parte de Ministerio del Ambiente como empresa con Punto Verde.

e) Compañía reconocida en el mercado entre las diez que mayoritariamente aplican la RSE frente al cambio climático por parte del Ministerio de Electricidad y Energía Renovable.

\subsection{Baviera S.A y RSE}

En el caso Colombiano se explicará la RSE realizada por la cervecería Baviera. Bavaria S.A es la cervecería más grande en Colombia y en el 2005 el Grupo Empresarial Bavaria y SABMiller PLC, firmaron un acuerdo de fusión (Plano, 2011). Bavaria desde hace 125 años ha estado implicada en la realización de proyectos sociales en favor de los colombianos. Sus principales proyectos se encuentran en inversiones sociales y ambientales a favor de las comunidades y grupos vulnerables y proyectos que contribuyen a la preservación del medio ambiente (Ruiz, 2014).

La Fundación Bavaria cuenta con un presupuesto mayor a $\$ 18.000$ millones para la ejecución de los proyectos sociales (Ruiz, 2014). Para cada uno de sus programas, Bavaria posee indicadores que cuantifican el impacto y el desempeño de cada una de sus acciones (Baez, 2015). Según estadísticas de la compañía mediante los programas ha logrado mejorar la calidad de vida de las familias, se ha creado empleo y se ha protegido más de 20.000 hectáreas de los ríos cercanos a donde realiza sus operaciones (Ruiz, 2014). Programas como "4e Camino al Progreso", "Zonas Libres de Pobreza" y "Destapa Futuro" han ayudado al desarrollo y crecimiento de comunidades y también a crear empresas que sean fuente de empleo (Ruiz, 2014).

El programa social estrella de Bavaria es el denominado "4e Camino al Progreso", el mismo que se encuentra aliado con el Banco Interamericano de Desarrollo y con FUNDES la misma que es una firma de consultoría, que lleva a los pequeños y medianos negocios al futuro (Haar, 2016). Este programa está destinado a tenderos emprendedores, que lideran el crecimiento de su negocio y a su vez el de su familia y comunidad (4e Camino al progreso). Bavaria busca que más de 17000 tenderos vulnerables puedan mejorar su calidad de vida y con ello la de su familia (Ruiz, 2014). 
Ruiz (2014) indica que los objetivos del programa se logran mediante clases y entrenamiento presencia, además de mentorías en habilidades para la vida y el liderazgo, así como el fortalecimiento de herramientas y conocimientos para el acceso a financiamiento y tecnología. Además expone que como meta a corto plazo Bavaria pretende mejorar la calidad de vida de 17.300 tenderos, ayudar a 2.500 familias para que salgan de la pobreza extrema, además se proyectan a ayudar a 7.500 empleados emprendedores para que mediante su trabajo transformen y dinamicen la economía colombiana.

\subsection{La Compañia de Cervecerías Unidad y sus programas de Responsabilidad Social}

Compañía Cervecerías Unidas S.a. se formó en 1902 con la unión entre la cervecera Plagemann, la fábrica de Cerveza Limache y la Fábrica de Cerveza y Hielo de Gubler y Cousiño. Convirtiéndose años más tarde en la empresa líder del negocio de cervezas en Chile (CCU). Las acciones y programas de Responsabilidad Social en la CCU se centran en dos principios. Primero, la operación en los negocios de CCU en sus bebidas alcohólicas está orientada un público mayor de 18 años. Como también que el consumo de alcohol debe hacerse de manera moderada y responsable ya que se vuelve perjudicial si se lo consume en exceso (CCU, 2014).

De manera que siguiendo estos principios se creó y desarrolló el programa denominado "Educar en Familia". Con el propósito de soportar y fortalecer el rol de los padres al momento de educarlos acerca del consumo responsable de alcohol.

Se implementa el programa promoviendo 5 actitudes para fomentar el diálogo entre padres e hijos (CCU):

a) "Predique siempre con el ejemplo"

b) "Hable con claridad y entre información precisa"

c) "Abarque el tema en familia"

d) "Acéptelo: usted es capaz

e) "Atento a las señales"

En las Fiestas Patrias celebradas cada 18 de septiembre junto al apoyo de la Policía (Carabineros) de Chile se reparte un folleto educativo por todo el país. En donde se recomienda tanto a peatones como conductores acerca de comportamiento seguro, mostrando estadísticas que le muestren el grado de las consecuencias. Así mismo, con el mismo propósito las marcas de CCU como Cristal continúan promoviendo por medio de propagandas las consecuencias de conducir bajo la influencia del alcohol de la cerveza.

\subsection{CCU y la cultura}

En el ámbito cultural CCU a promovido programas como "CCU y la Cultura" y "Cerveza Cristal en el arte" para apoyar al arte contemporáneo chileno. De tal forma que se aproxime la comunidad a actividades de museos y galerías de Santiago al promover el arte como una actividad que transmite valores positivos. La campaña se inició en 1993 y se ha mantenido hasta la actualidad apoyando a artistas chilenos de forma permanente, facilitando el acceso de sus obras a exhibiciones de galerías importantes como el museo de bellas Artes y el museo de Arte Contemporáneo de Santiago (CCU, 2014).

\subsection{CCU y el medio ambiente}

La CCU ha desarrollado un programa de Gestión Ambiental que permite imponer metodologías para la protección del medio. Las cuales están inmersas en los compromisos de la empresa. Este incluye el manejo de residuos sólidos, líquidos y gaseosos, resaltando la importancia de su cuidado con el propósito de perpetuar un ambiente apto para las generaciones futuras. Como por ejemplo su programa "Cuidemos nuestro ambiente" en el cual se cultiva 100,000 árboles aproximadamente como donación a la comunidad (CCU, 2014).

\section{Conclusiones}

En este artículo se ha presentado los avances y retrocesos en materia de RSE de forma regional en Latinoamérica y específico en Ecuador observando que hay una profunda vinculación entre la organización y los stakeholders como generadores del desarrollo económico, social y ambiental; permitiendo mejorar sus estilos de vida y construyendo a una sociedad saludable y de progreso. Sobre la base de los estudios efectuados podemos determinar que el éxito de una organización es proporcional a su actuación y preocupación por su gente, su comunidad, su cadena de valor en general y su ecosistema sin dejar en segundo plano; la finalidad económica de prosperidad, crecimiento y rentabilidad que toda empresa busca. Este ejercicio nos lleva a notar, una realidad no complaciente para Ecuador porque refleja una práctica no habitual y no universal en temas de responsabilidad social empresarial revelándose únicamente casos muy aislados de implementación de RSE que en su mayoría provienen de corporaciones multinacionales.

\section{Referencias}

[1] 4e Camino al progreso. (s.f.). Camino al progreso. Obtenido de HAY RAZONES DE PESO QUE NOS INSPIRAN A CREAR HISTORIAS DE PROGRESO: http://www.caminoalprogreso.com/ 
[2] Alonso, V. (2006). Avances en la discución sobre la ISO 26000 en América Latina: antecedentes para apoyar el proceso ISO en la Región. CEPAL.

[3] Altvater, E., \& Mahnkopf, B. (2002). Las limitaciones de la Globalización. Buenos Aires: Siglo XXI Editores Argentina S.A.

[4] AVINA, \& Korin, M. (2011). EN BUSCA DE LA SOSTENIBILIDAD: El camino de la Responsabilidad Social Empresarial en América Latina y la contribución de la Fundación AVINA. Buenos Aires: Fundación AVINA.

[5] Baez, X. (2015). Responnsabilidad Social en Bavaria S.A. Universidad Militar Nueva Granada.

[6] Bangochea, A. (2010). Dimensión Medioambiental de la RSC. España: Netbiblo.

[7] Carneiro, M. (2004). La resposabilidad social corporativa interna: la nueva frontera de los recursos humanos. Esic Editorial.

[8] Casado, F. (2006). La RSE ante el espejo: Carencias, complejos y expectativas de la empresa responsable en el siglo XXI. España: Prensas Universitarias de Zaragoza.

[9] Cerveceria Nacional. (23 de 09 de 2014). Cerveceria Nacional. Obtenido de http://cervecerianacional.ec/empresa.php

[10] Cervecería Nacional S.A. (2011). Informe CN: Haciendo la Diferencia 2011. Guayaquil.

[11] Cervecería Nacional S.A. (21 de 09 de 2014). www.cervecerianacional.ec. Obtenido de http://www.cervecerianacional.ec/desarrollo-

sostenible.php

[12] Cooper, S. (2004). Corporate Social Performance: a Stakeholder Approach. Burlington: Ashgate.

[13] Corrales, M. L. (2011). La Responsabilidad Social Empresarial en la pequeña y mediana empresa. España: Consulting.

[14] Correa, M., Flynn, S., \& Amit, A. (2004). Responsabilidad social corporativa en América Latina: una visióm empresarail. Santiago de Chile.

[15] Deloitte. (2014). Informe de Percepción de la Responsabilidad Corporativa y Sostenibilidad. Quito.

[16] Ekos Negocios. (2012). Cerveceria Nacional:10 Prioridades para un modelo sostenible. Guayaquil.

[17] Ekos Negocios. (2013). Reutilización de materiales. Guayaquil.

[18] Fernandez, R. (2010). Responsabilidad Social Corporativa. Editorial Club Universitario.

[19] Fernández, R. (2011). La dimensión económica del desarrollo sostenible. España: Editorial Club Universitario.
[20] Fuertes, F., Lara, M., \& Kosacoff, B. (2006). La responsabilidad social empresaria: sólo un discurso. Santiago de Chile: CEPAL.

[21] Guibert, J. (2009). Responsabilidad Social Empresarial: Competividad y casos de buenas prácticas en pymes. San Sebastian: Universidad de Deusto.

[22] Gutierrez, R., Avella, L., \& Villar, R. (2006). Aportes y desafíos de la responsabilidad social empresal en Colombia . Colombia: Fundación Corona.

[23] Haar, J. (20 de 03 de 2016). Bavaria. Obtenido de ¿Cómo una empresa incorpora la responsabilidad social corporativa?: http://www.bavaria.co/prensa/noticias/c\%C3\%B3mouna-empresa-incorpora-la-responsabilidad-socialcorporativa

[24] Jáuregui, R. (2008). América Latina, España y la RSE: Contexto, perspectivas y propuestas. Madrid: Fundación Carolina.

[25] Mendez, F. (2007). La Comunicación Organizacional como gestora del conocimiento y la Responsabilidad Social. Razón y Palabra.

[26] Morales, K. (2012). Sostenibilidad Empresarial: Experiencias de Responsabilidad Social en Empresas Ecuatorianas. Quito: Disclaimer.

[27] Navarro, F. (2012). Responsabilidad social corporativa. Madrid: ESIC Editorial.

[28] Payne, S., \& Calton, J. (2004). Exploring research potentials and applications for Multistakeholders. Holanda: Academic Publisher.

[29] Plano, D. (01 de 08 de 2011). Banco de la República Actividad Cultural. Obtenido de La industria cervecera en Colombia: http://www.banrepcultural.org/blaavirtual/revistas/crede ncial/agosto2011/cerveza-industria

[30] Riechmann, J., \& Naredo, J. (1995). De la economía a la ecología. Madrid.

[31] Roser, I. (2005). Guía de RSC para PYMES . España: Fundación El Monte.

[32] Ruiz, L. (04 de 06 de 2014). La República. Obtenido de Bavaria a través de su fundación apoya a las víctimas de la violencia y sus familias: http://www.larepublica.co/bavaria-trav\%C3\%A9s-de-sufundaci\% $\% 3 \% \mathrm{~B} 3 n$-apoya-las-v\%C3\%ADctimas-de-laviolencia-y-sus-familias_129866

[33] Vives, A. (2008). El papel de la RSE en América Latina:Diferente al de Europa. España: Fundación Carolina. 